\section{This strange Universe}

John Peacock

Basics of Modern Cosmology. By A. D. Dolgov, M. V. Sazhin and Ya. B. Zeldovich. Editions Frontières: 1991. Pp.247. Hbk, $\$ 40 ; p b k, \$ 28$.

More than three years after his death, a new book has appeared bearing the name of one of this century's greatest physicists. Yakov Borisovich Zeldovich (1914-1987) was a man of almost unequalled breadth of interests, who made fundamental advances in gas dynamics, particle physics and cosmology. Many who never had the privilege of meeting him (including myself, sadly) have got to know the Zeldovich style through his monumental two-volume works on gas dynamics (with Y. P. Raizer) and relativistic astrophysics (with I. D. Novikov), together with innumerable research papers. These writings are characterized by an admirable directness: Zeldovich usually goes straight to the heart of a problem, rarely getting distracted in the erection of unnecessary formalism. Some of his most influential papers are almost unbelievably short. The impression that emerges is of a scientist equipped with great depth of insight, and not someone likely to have had much patience with the bureaucratic society in which he lived and worked. Some of these features emerge from the obituary by Andrei Sakharov (see Nature 331, $671 ; 1988)$, but not the story I like best: of Zeldovich cannily protecting himself from police harassment by donning his medals before going out and getting drunk.

Basics of Modern Cosmology thus has a rather high standard to live up to, and I have to say I found it a little disappointing by comparison with the expectations it raised. It is a translation of a book originally completed in 1986 , and based upon lectures given by Zeldovich, although more than two-thirds of the text was written by A. D. Dolgov and M. V. Sazhin. The aim is to give an introduction to the revolution in cosmology that occurred in the first half of the 1980 s, caused by the ingestion of methods and ideas from particle physics. The book can be divided into four parts: (1) principles of pre-inflation cosmology; (2) a primer on particle physics; (3) a discussion of scalar fields and inflation, including baryosynthesis and generation of fluctuations; (4) the large-scale structure of the Universe. This is an awful lot of material to fit into 247 pages, especially given that the book tries to be very complete. The result is, inevitably, that most topics are treated rather briefly. This is a pity, given that the amount of introductory material indicates that the book is probably intended for students unfamiliar with modern cosmology. Such

readers will be frustrated by the many formulae that are pulled out of the air without derivation or reference, particularly where these relate to items of technical difficulty very much greater than the surrounding material (such as the casual use of the path integral over euclidean metrics in chapter 9). Novices in non-Soviet countries will also be

\section{IMAGE UNAVAILABLE FOR COPYRIGHT REASONS}

\section{Zeldovich - equipped with great insight.}

hampered by the fact that the terminology often fails to connect with that common in Western research literature. Nothing wrong with that in principle: the fact that most of the book's references are to Soviet literature is a valuable antidote to the all-too-common belief that all new developments in cosmology appear in the Astrophysical Journal. But this approach has been carried too far if it means that the spectrum referred to in the West as 'Zeldovich', ' $n=1$ ' or 'scale-invariant' (one of Zeldovich's most important bequests to cosmology) is apparently not mentioned, unless you know what you are looking for. It is quite possible to give the Soviet view of the subject without being obscure, or using a widely varying level of difficulty, as is shown in the recent excellent text by A. Linde (Particle Physics and Inflationary Cosmology, Harwood, 1990).
So, if this is not the place to send an illequipped graduate student, what will the established professional researcher find here? Several aspects of the book bear the strong imprint of Zeldovich's views on the subject, which are sometimes at variance with Western orthodoxy, and make for thought-provoking reading. Zeldovich always placed great weight on the fact that the energy of a closed Universe is zero; the idea that the 'spontaneous birth' of a closed Universe does not violate energy conservation and therefore might be preferred in a quantum sense is treated quite extensively. Also, a whole chapter is devoted to A. A. Starobinsky's ideas on inflation being driven by vacuum polarization (that is, quantum F corrections to the energy-momentum tonsor) rather than by a scalar field. ${ }^{2}$ This alternative is subsequently dropped without explanation, however, and the book then proceeds along more standard lines. This is a pity, as I feel an opportunity was lost for taking a more critical look at some of our current ideas. In particular, the crucial defect of inflationary cosmology as it stands is that the scalar field must be very weakly coupled, to avoid the overproduction of inhomogeneities. Unlike Guth's original proposal, its existence is thus not at all well motivated from particle physics. This weakness deserves to be seized on, especially by a book that is not afraid of being a little heterodox, but it passes almost without comment.

The last two chapters are on fluctuations and large-scale structure, and I particularly looked forward to seeing Zeldovich's last views on a field he helped to create. In the end I was disappointed, especially because very little is said about confrontation with observations. Perhaps the authors consider this to be unnecessary or uninteresting: "The formation of the large-scale structure of the Universe is already understood qualitatively and only the quantitative details need to be worked out." This may well be true in the sense that brain surgery is only applied quantum mechanics, but it does give a rather peculiar view of the subject. One of the most exciting aspects of cosmology in recent years has been the rapid accumulation of evidence about the structure of the Universe we inhabit. Different researchers will disagree about whether this process is converging to any sort of understanding, but it is undeniably a source of great intellectual enjoyment. A book on this subject, especially one for students, should be able to communicate the sheer excitement of being able to understand for the first time the main features of this strange Universe. Sadly, this one does not.

John Peacock is at the Royal Observatory, Blackford Hill, Edinburgh EH9 $3 \mathrm{HJ}$, UK. 